\title{
The development and evaluation of Robot Light Skin: a novel robot signalling system to improve communication in industrial human-robot collaboration
}

\author{
Gilbert Tang, Phil Webb, John Thrower \\ Cranfield University \\ Corresponding author: Gilbert Tang, g.tang@cranfield.ac.uk
}

\begin{abstract}
In a human-robot collaborative production system, the robot could make request for interaction or notify the human operator if an uncertainty arises. Conventional industrial tower lights were designed for generic machine signalling purposes which may not be the ultimate solution for robot signalling in a collaborative setting. In this type of system, human operators could be monitoring multiple robots while carrying out a manual task so it is important to minimise the diversion of their attention. This paper presents a novel robot signalling solution, the Robot Light Skin(RLS), which is an integrated signalling system that could be used on most articulated robots. Our experiment was conducted to validate this concept in terms of its effect on improving operator's reaction time, hitrate, awareness and task performance. The results showed that participants reacted faster to the RLS as well as achieved higher hit-rate. An eye tracker was used in the experiment which shows a reduction in diversion away from the manual task when using the RLS. Future study should explore the effect of the RLS concept on large-scale systems and multi-robot systems.
\end{abstract}

Keywords - Human-robot interaction, collaborative system, industrial ergonomics, automation

\section{Introduction}

The use of industrial robots has been continuously increasing to meet rising global demands in manufacturing output and quality as well as reducing operating cost [1]. While most of these robots are designed to perform fully automated routine operations within caged areas, the recently introduced collaborative robots are designed to function alongside human operators and in some cases cooperate with them. Collaborative systems are offered as semi-automated solutions to manufacturing applications where there are variability in the components, procedures or operating environment that may require manual input from human operators $[2,3]$. These systems can provide the cognitive flexibility of human workers and the repeatability and robustness of industrial robots. In collaborative systems, humans and robots are sharing workspaces and often one human operator can be managing multiple robots while carrying out other tasks. In this case, robots must communicate with the operator effectively to maintain safe and seamless interactions.

In conventional industrial settings, tower lights are commonly used for visual signalling of factory production cells. Small assembly lines or cells are often operating in parallel with one tower light placed above the last station along the transportation aisle indicating the state of operation. In the context of visual management, tower lights are known as Andon lights, which support lean production systems as a means of indicating machines' status; one of the early adopter was the Toyota Production System [4,5]. Andon light is one aspect of the Jidoka manufacturing principle which refers to the practice of stopping work immediately when a problem occurs. It can provide visual signals to indicate the present of wastes in a factory, which are often the main source of 
potential improvements in business performance. An effective visual signalling system ensures that line status could be observed at a glance which then enable operators to alert relevant personnel for assistance $[6,7,8]$.

Industrial tower lights are based on a simple system that is used in everyday life: the traffic light system. The main benefit of tower lights with red, yellow and green lights is a simple and effective communication tool which allows factory managers and supervisors to be aware of the state of production lines at a glance [9]. The intention is to enable users to perceive machines' state without the requirement for significant cognitive workload which will speed up the users' response time to the signals as well as reserving mental capacity to focus on other tasks. Apart from traffic signals and factory systems, the traffic light concept is also used in other ordinary applications. For example, in supermarket self-checkout each counter is equipped with one tower light which is usually positioned above the machine to indicate whether it is in normal working order or when attention is needed. In the event if an error occurs, the tower light changes from green to red to indicate human help is needed, and a contact staff will approach the machine when they notice the signal. In this case, the response time of the contact staff is largely dependent on the ease of noticing this visual signal $[10,11,12]$. Despite the evidential advantages shown in conventional factory systems, tower lights were not design specifically for human-robot collaborative applications so their efficacy can be significantly reduced. For instance, the positioning of tower light is restricted in a way that should not hinder the movement of the robot. On the other hand, the distanced light signals can divert operators' attention away from the robot and the manual task. Thus, the main aim of our study is to explore the key impacts of an alternative indication system on human-robot collaboration.

Human-machine interface developments are often technology-focused, where display of data in these systems is designed solely based on technical specification; it is often arranged in a way that is not ideally suited to support human tasks. Using such approaches could result with negative effects on the user performance, which can cause design-induced errors [13]. By applying the philosophy of user-centred design throughout the system design cycle, an effective collaborative system is achievable. A user-centred design challenges designers to build human-machine interfaces around the capabilities of the potential users. It also improves user acceptance and satisfaction as a side benefit [14]. When designing a communication system for human-robot collaborative working, it is important to consider human perception of signals in order to develop an effective system. For instance, in an industrial human-robot collaboration scenario, an operator could be carrying out a manual task whilst monitoring one or more robots. In a collaborative working environment, a robot could make requests for interaction to complete a task jointly with an operator, or when an abnormality is detected an intervention would be required. The robot will signal to the operator and must catch the operator's attention, who might be focused on a different task at that point.

Humans in general have limited capacity in paying attention to multiple events due to their information processing bottlenecks, people withdraw from some tasks in order to collaborate effectively with others [15]. Industrial tower lights are typically fixed at a location near the machine, and operators have to draw their visual attention to the light unit to detect any changes of light. This is an example of top-down spatial attention where the subject can focus on a small region of space within their field of view based on internal guidance of attention based on prior knowledge and current goals [16]. Thus, extra mental capacity is needed to extract useful information which could lead to higher than necessary workload and error. Humans react involuntarily to salient events as well as pay attention to objects which are relevant to their current activity. Visual attention is highly affected by the salience of objects (i.e. their size, colour, location) which can be defined as bottomup attention $[17,18]$. Furthermore, research evidences have shown that bottom-up attention is 
faster than top-down attention due to it being an automatic reaction triggered by sensory information through the brain [19-25]. In this case, it is logical to minimise the number of objects which require visual attention within a collaborative robotic cell. Thus, the signalling interface should be an integrated system on the robot to provide dynamic visual signals which can be captured by users with ease using bottom-up attention.

Reaction time (RT) is another important aspect to take into consideration in this research because every second of system downtime could be costly for any production line. Therefore, a human-robot interface must be able to deliver signals to users as quickly as possible. Murray and Caldwell reported significantly longer RTs as the number of displays and display figures to be monitored increased, which supports the Hick's Law where decision time increases with the number of choices $[26,27]$. Humans' reaction to signals can also be delayed as an effect of increases in RT as viewing angle increased [28], as well as background noise [29] and task complexity [30]. Based on the limitation of viewing angle and position of traditional stack lights, users may have to rely on their peripheral vision to receive the light signal. However, several studies have shown that it usually takes longer for people to notice abnormalities in their peripheral vision [31,32]. This further supports the theory that visual signal should be within proximity of the area of interest for the particular application.

Majority of studies carried out on human-robot interaction through visual signalling are in the context of social and mobile robots, but little work has focused on industrial human-robot collaborative system [33]. Thus, the aim of this research is to develop and evaluate a visual signalling system concept specifically designed for collaborative production system which enables the operator to react promptly to a robot signal during a manufacturing operation.

The rest of the paper is organised as follows: section 2 describes the proposed signalling system concept. Section 3 describes the methodology for validation of the concept and section 4 discusses the experiment results. Finally, section 5 concludes the paper with suggestions made for future work.

\section{Robot Light Skin}

\subsection{The proposed solution}

This paper proposes the Robot Light Skin (RLS) concept as a visual indication system which communicates robots' status to human operators. The device will cover the upper arm and wrist area of articulated robots, and it illuminates in different colours to indicate the state of the robot as illustrated in figure 1. In this case, the robot becomes the source of the signal and unlike conventional indication systems; it does not create another distraction for human workers. This enables operators to response to interaction requests from corresponding robots promptly and accurately as illustrated in figure 2 . This concept could be realised using organic light-emitting diode (OLED) or light-emitting diode (LED) array, both are flexible and fully programmable which could be used to cover the exterior of an industrial robot as a signal light or for displaying text form status. However, the cost of OLED light sheet is relatively high compared to other viable lighting technologies such as fully programmable LED light strips. The cost of this technology should eventually decrease from the current level which enables it to be a feasibly low cost solution. Furthermore, the developed concept will be relatively simple to implement onto collaborative systems or retrofitted to existing production systems when compared with other interactive technologies such as Virtual Reality (VR) [42, 43] and Augmented Reality (AR) [39, 40]. It can also be integrated with other interactive devices such as tactile sensing modules [41] or combined with other safety strategy such as collision avoidance [44]. In terms of practicality for deployment, the 
size of the RLS could be made adjustable for attaching to different robots, and the RLS should not cover the joints or motors to prevent restrictions of the robot movement or heat dissipation. For the development in this research, flexible LED light strip was used for the early phase validation of the concept.

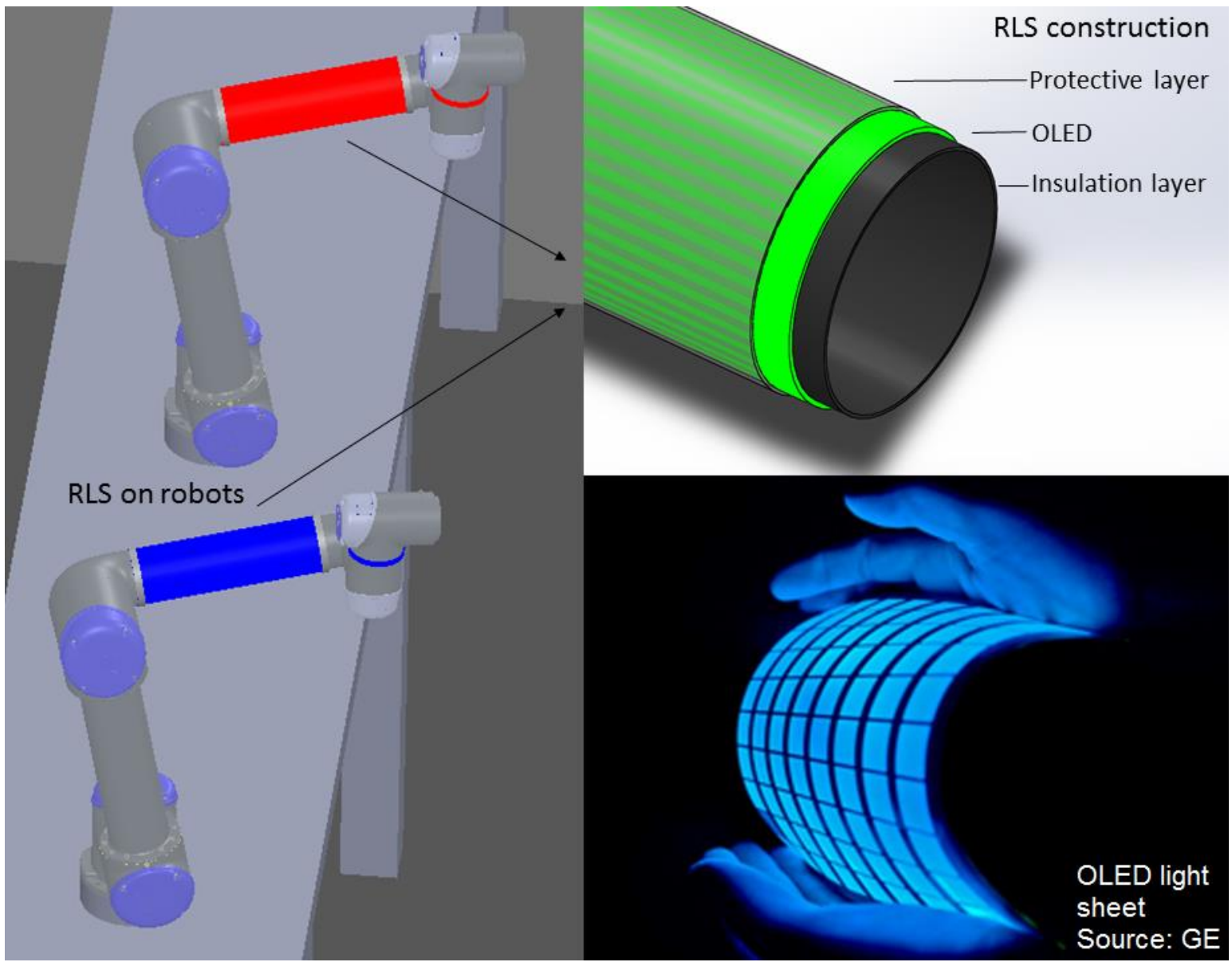

Figure 1 - application of RLS on robot and its construction

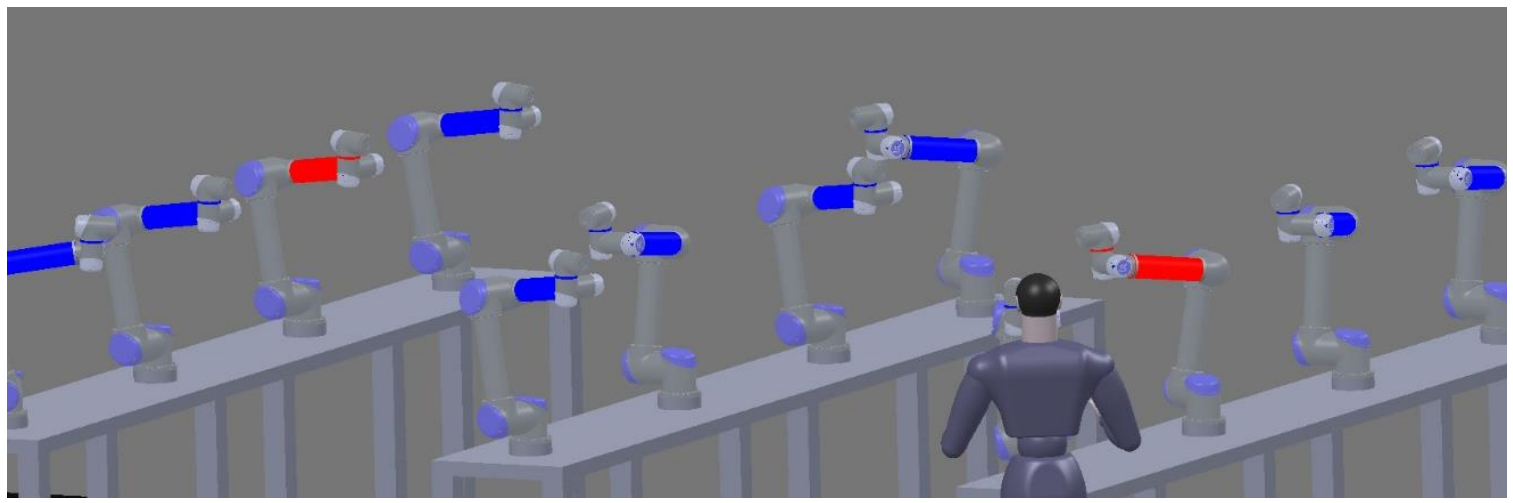

Figure 2 - an illustration of how RLS could assist operator spotting robots that require attention

\subsection{Concept validation}

An exploratory experiment was conducted to validate the concept by comparing its effectiveness against a tower light. Its aims are to ascertain whether the robot indicator design concept compared to an industrial tower light increases awareness, reduces workload and improves reaction times for operators. The objectives are to evaluate the performance of participants completing an assembly 
task when subject to different experimental conditions, measure participants' reaction times to different type of signal lights while observing the robot performing a task, and to measure the visual fixations of participants throughout the task. The experimental parameters were measured with an eye tracker device. The results of this experiment will be used to make suggestions for system refinement and future development.

\section{Method}

\subsection{Participants}

Sixteen people from the general population of Cranfield University participated in the experiment. 12 of them were males and four of them were female, and all participants were right-handed. Their age ranged from 23 to 56 years (Mean=30, $S D=9.88$ ).

\subsection{Design}

This experiment followed a repeated measures within-subjects $2 \times 2$ design. The two independent variables are signal light type and participant's standing position relative to the robot. There were two signal light types: RLS and tower light (TL), and two standing positions: forward and side (relative to the robot). Participants had to complete one time in each of the four conditions which are combinations of different signal type and standing position of participants, which are (RLS: forward), (RLS: side), (TL: Forward) and (TL: side). The four conditions were counterbalanced to eliminate order effect. A number of dependant variables were measured which include reaction time, hit-rate, task performance, ease of monitoring and visual fixation and dwell. Each experiment took around 35 minutes.

\subsection{Experimental cell}

The experiment took place in a $3960 \mathrm{~mm} \times 3900 \mathrm{~mm}$ laboratory area surrounded by 4 sides of wall. The robot arm was positioned on top of a stand at a height of $1030 \mathrm{~mm}$. The robot has $850 \mathrm{~mm}$ reach with a circular working envelop. A $1220 \mathrm{~mm} \times 800 \mathrm{~mm}$ robot worktop with a height of $920 \mathrm{~mm}$ was positioned next to the robot base with a $200 \mathrm{~mm}$ clearance. Six sets of pipe holders were presented on the robot worktop for the robot to perform the pick and place task. A manual workbench with surface area of $630 \mathrm{~mm} \times 785 \mathrm{~mm}$ was attached to the robot worktop at the same height. A button was positioned on the manual workbench which was connected to a National Instrument logging system to record participants' reaction time and hit-rate of reacting to light signals. Three part trays were also presented on the workbench which contain component for the manual assembly task. Both worktops had a matt surface which minimised reflection of the indication lights.

An industrial tower light was positioned at a height of $1210 \mathrm{~mm}$ in a location that was visible to participants in both test positions without any obstruction from robot movements. A LED light strip was wrapped around the robot which covers the area between the elbow and the wrist, the wrist and the base, to represent the RLS concept as shown in figure 3, 4 and 9. Both the tower light and RLS were positioned similar to current industrial placement and accordingly to British Standards for indication systems for industrial machinery [34,35]. The experiment area had no direct sun light and the lighting level was kept constant at 400lux throughout the entire experiment. The experiment setup is shown in figure 3 . 


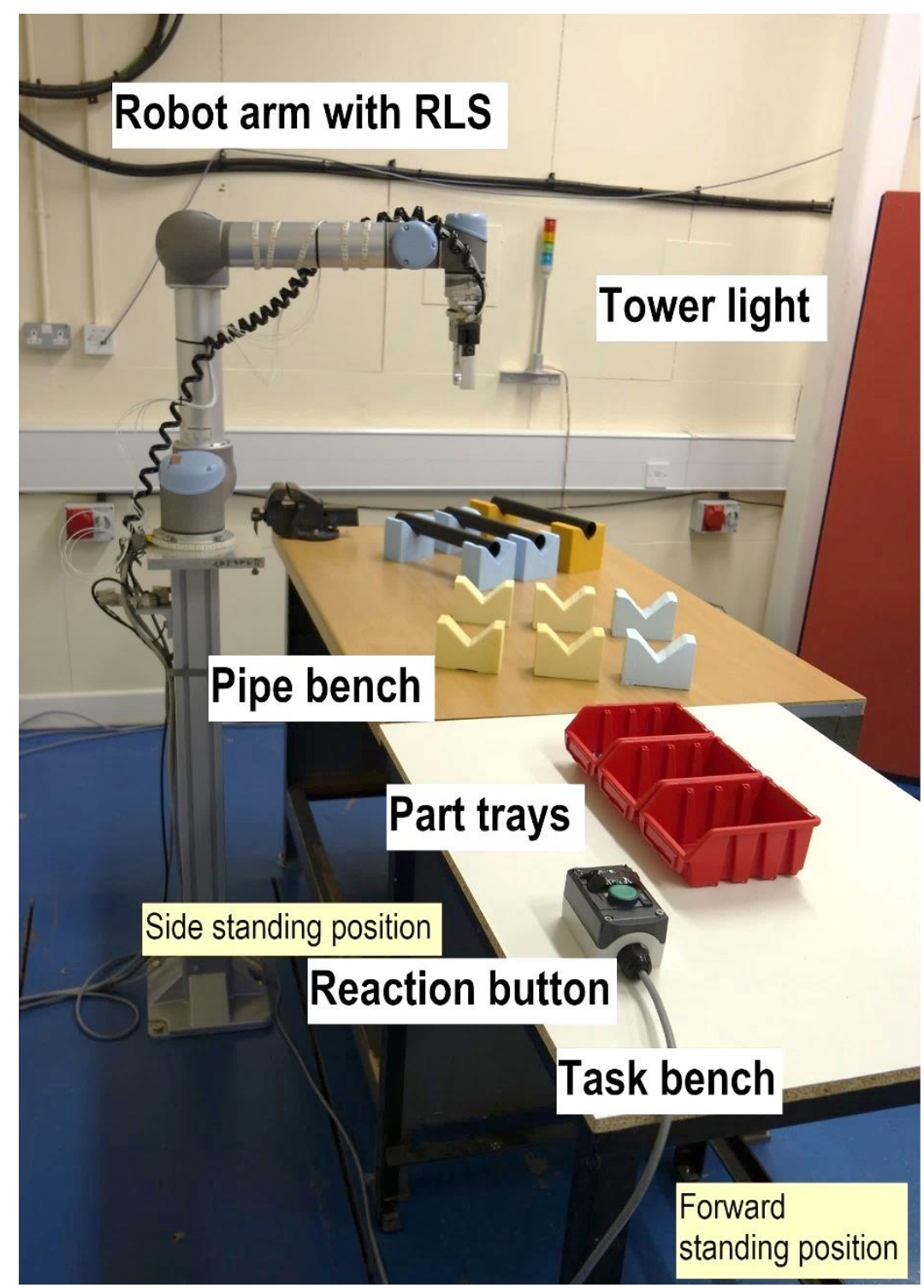

Figure 3 - showing the experiment setup and positions of different components

\subsection{Eye tracking}

The participants' eye movements were recorded using a mobile head-mounted eye-tracker (SensoMotoric's BeGaze $@$ eye tracking system). The device is portable which enables participants to move their head naturally without limitation during the task. It is shaped similarly to safety glasses which simulate the physical restrictions in real working condition when safety wears are worn [36]. The reasons for using this equipment are to ascertain common points of interest in different scenarios, and the effect of using the developed signalling method. Thus, the hypothesis can be verified and the data can also support other findings. This eye tracking equipment consists of glasses with in-built cameras that track human eye pupil activity while simultaneously recording field of vision. All participants wore the equipment throughout the experiment as illustrated in figure 4. The tracking data was analysed using BeGaze software utilising Area of Interest (AOI) semantic gaze mapping. The AOls mapped include the manual task work top, robot work top, robot (with and without light on), and the tower light (on and off). Fixation time and dwell time measurements were mapped to corresponding AOls using the BeGaze software where dwell time is the total of fixation durations and saccade durations that hit the AOI. 


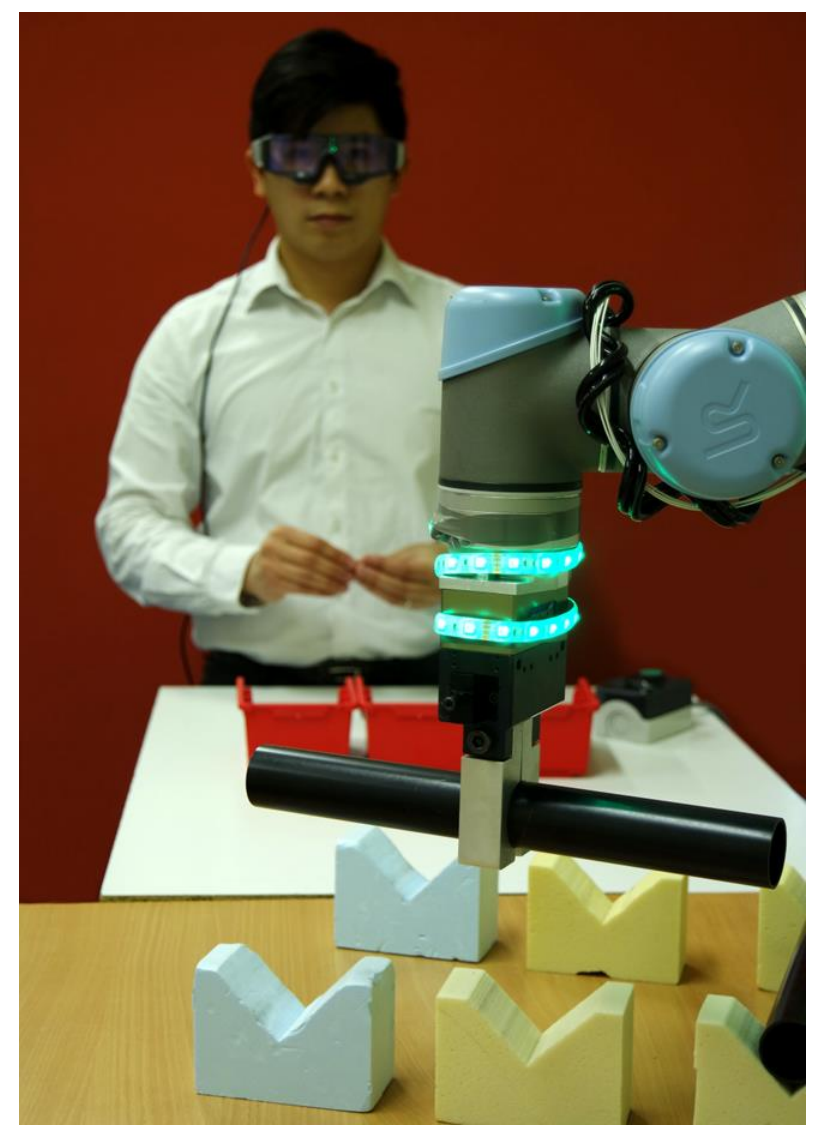

Figure 4 - a participant wearing an eye tracker while performing the assembly task and a robot with a lit RLS device carrying out a pick and place operation

\subsection{Procedure}

Informed consent was sought from participants prior to participating in the experiment. Participants were informed regarding their right to withdraw at any time, confidentially and anonymously. They were asked to provide their demographic information (age, sex, job title) in a questionnaire. A scripted experiment briefing was given to each participant to explain about the purpose of the study and the expectations of him or her. Participants were led into the experimental cell and they were fitted with the head-mounted eye-trackers.

Participants were invited to complete a pre-experimental task in order to familiarise themselves with the assembly task and minimise the practice effects, which lasted for three minutes. This assembly task involved inserting a washer onto a bolt, and then threading a nut to the bolt until the end of the bolt is flush with the washer. Upon satisfactory performance of the pre-experimental task participants completed a benchmarking exercise where their time spent on completion of five sets of assembly was measured. This measured time was divided by five to use as a benchmark time $b_{n}$ for calculation of the performance index from test result using (1).

$$
\rho=\frac{T}{b_{n}}
$$

$T$ is the time to complete a single assembly during the experiment sequence, and $b_{n}$ is the benchmark time to complete a single assembly. 


\subsection{Task}

Participants completed a simple assembly (nut, bolt and washer) task, while a robot completed a pick and place task nearby (figure 4). They were instructed to complete as many of the assemblies as possible while observing the robot task for any unusual manoeuvres and take mental note of each event within each experiment segment. The robot was programmed to carry out the same pick and place routine throughout the experiment. Each participant was asked to react by pressing a button in front of them when they saw a green or a red light on the robot or the tower light. Each participant had to operate in both a forward and side position in respect to the robot worktop orientation.

The experiment segments must be carried out enough times to collect an adequate amount of data as well as for counterbalancing results. However, due to the repetitive nature of the task it was important to restrict the length of the experiment and number of segment to minimise tediousness. As Walters et al have demonstrated, a long-term Human-Robot Proxemics study with repetitive experimental procedures can cause boredom which ultimately lead to early exit of participants [37]. This highlights restrictions of keeping experimental conditions controlled, and the necessity of planning realistic, engaging and varied experimental scenarios. Four pilot studies were carried out prior to the actual experiment to make refinements to the experimental process. No major changes have been made apart from the wording of questionnaires.

A semi-structured interview was carried out to gather subjective responses to the stimulus after each 3-minute segment with closed-ended questions regarding the ease of monitoring the light, observation of unusual robot movement, and level of tiredness. A final questionnaire with openedended questions was conducted to gauge relevant effects of different settings include perceived difficulty of the task, preference on the type of signal light, difficulty in robot observation, comfort issue in relation to wearing eye-tracker, and other issue caused by the eye-tracker. All participant responses were recorded using a questionnaire form. Their task performance, visual fixations, and their reaction times to the light signals were measured using different means.

\section{Results}

\subsection{Reaction time}

The results show that it takes longer for participants to react to light signals from the tower light source as shown in figure 5. A two-way repeated measure ANOVA with a Greenhouse-Geisser correction showed that mean reaction time differed significantly between light signal type $[F(1,15)=6.226, p=.025]$, but no significant difference was found in standing position $(p=.519)$. 


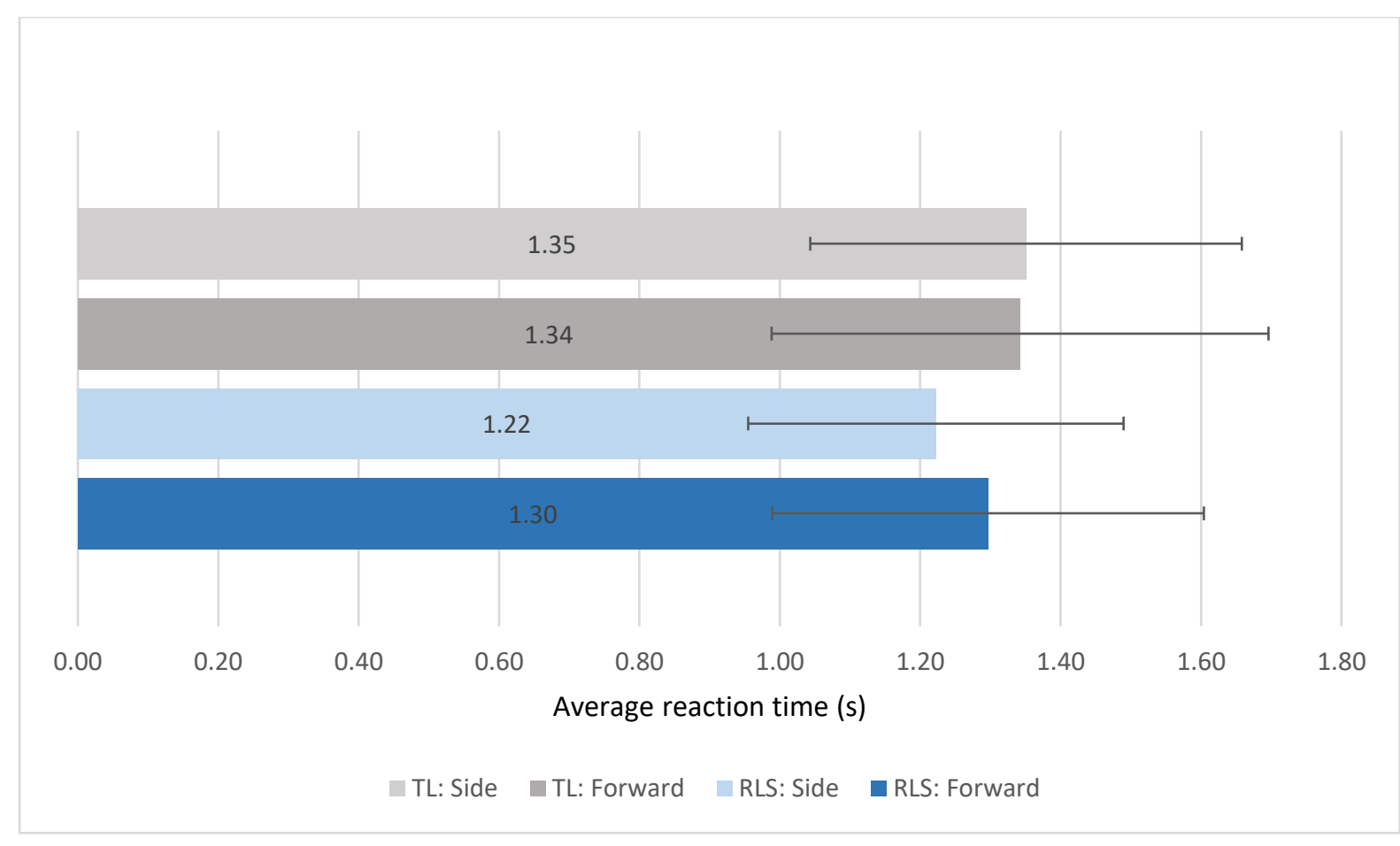

Figure 5 - mean reaction times of all participants in different scenarios

\subsection{Hit-rate}

Each signal light was on for three seconds and the participants were permitted to react to the signal by pressing the button, if participants fail to react within three seconds it will count as a miss even if the button is pressed. The difference in number of misses between the two light sources is significant as shown in figure 6 . The integrated robot light has received a total number of five misses in both the forward position and side position, whereas the tower light has 21 misses in forward position and 32 misses in side position. A two-way repeated measure ANOVA with a GreenhouseGeisser correction was used to analyse the overall light signals hit-rate and no statistical significance was found in the signal type $[F(1,15)=4.192, p>.05]$ or the standing position $[F(1,15)=2.249, p>.05]$. 


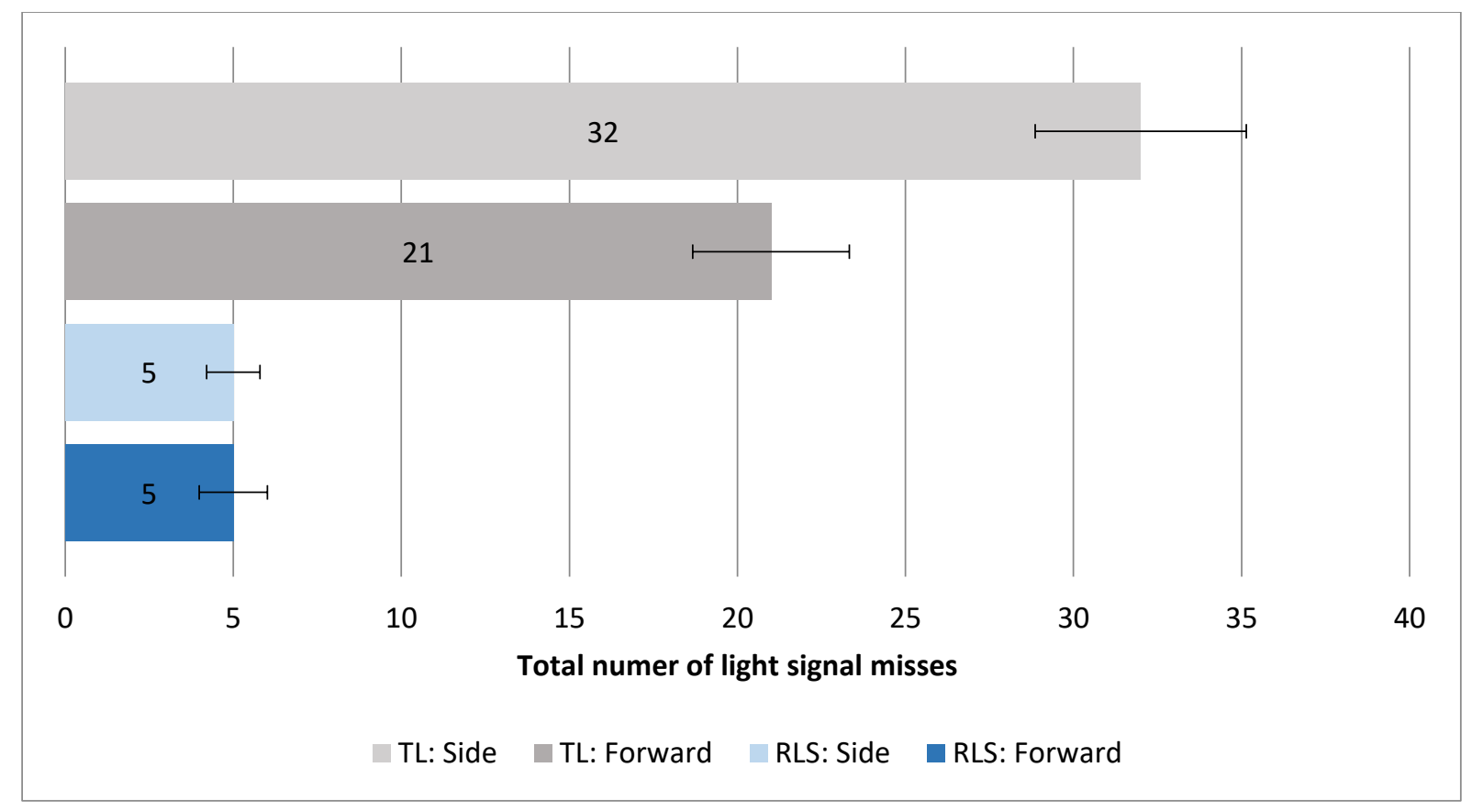

Figure 6 - combined hit rate for all participants

\subsection{Task performance}

The task performance is measured by comparing the completed assembly count at the end of each experiment segment to a benchmark as explained in method. A performance index is calculated for each segment using formula (1). A two-way repeated measure ANOVA with a Greenhouse-Geisser correction revealed no effect on performance index between signal type $[F(1,15)=1.494, P>.05]$ or standing position $[F(1,15)=3.378, P>.05]$ (figure 7 ).

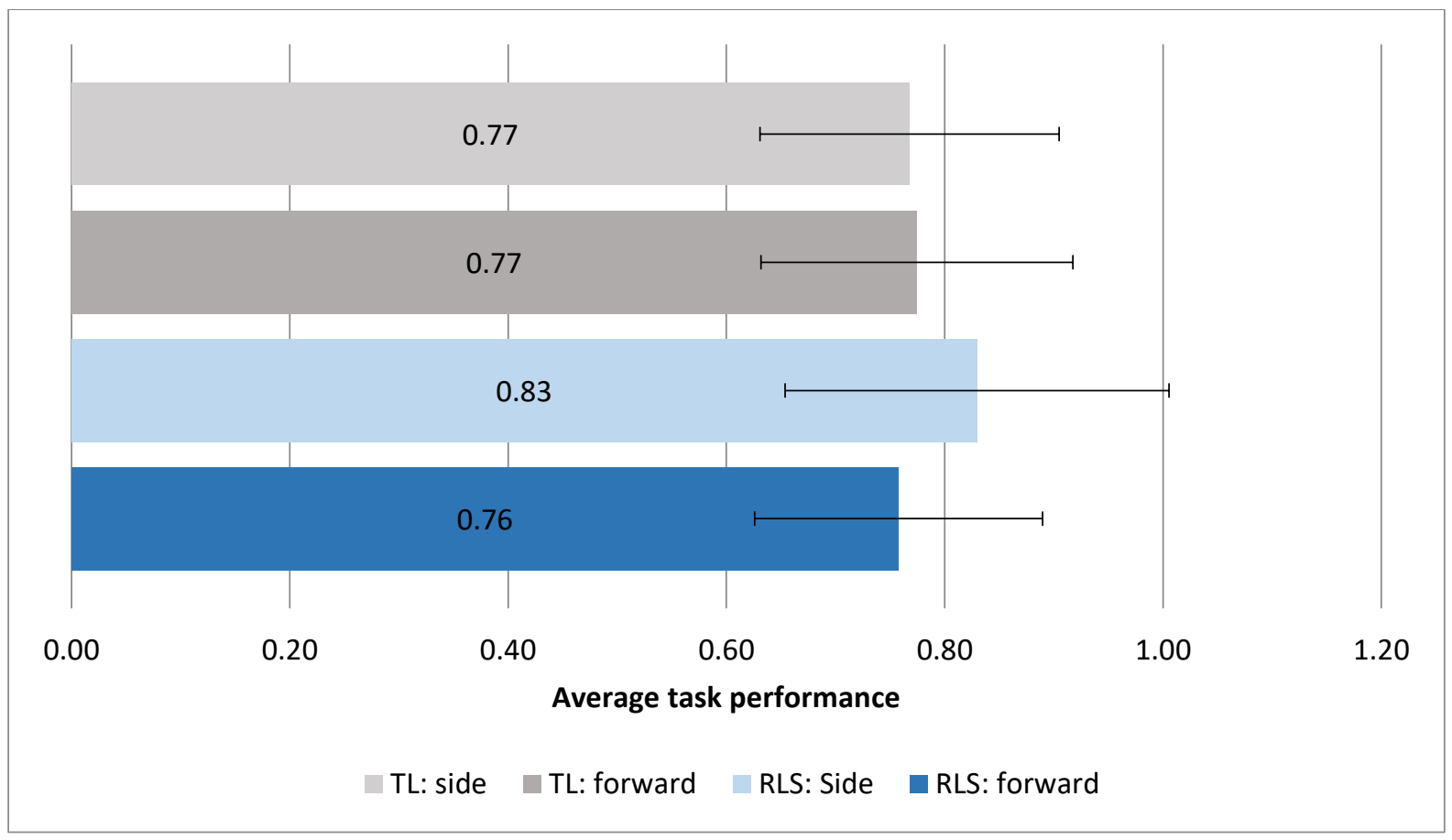

Figure 7 - no significant difference was found in the average task performance, which can be due to low demand manual task 


\subsection{Ease of monitoring}

After each experimental segment a semi-structured interview was carried out to receive feedback from participants. One of the questions was to score the ease of monitoring the signal light. A 7point Likert scale was used, where seven is the easiest and one is the most difficult. Participants found it easier to monitor signal lights in the forward position than when standing on the side position. The RLS received higher scores than TL when comparing the two devices in the same standing position as shown in figure 8. The RLS: side scored higher then TL: side but lower than TL: forward, and this can be due to the increased difficulty of monitoring light signals outside of the participants' peripheral.

A two-way repeated measure ANOVA with a Greenhouse-Geisser correction was used to assess the result which revealed significant effect in both signal type $[F(1,15)=7.716, p=.014]$ and standing position $[F(1,15)=10.363, p=.006]$.

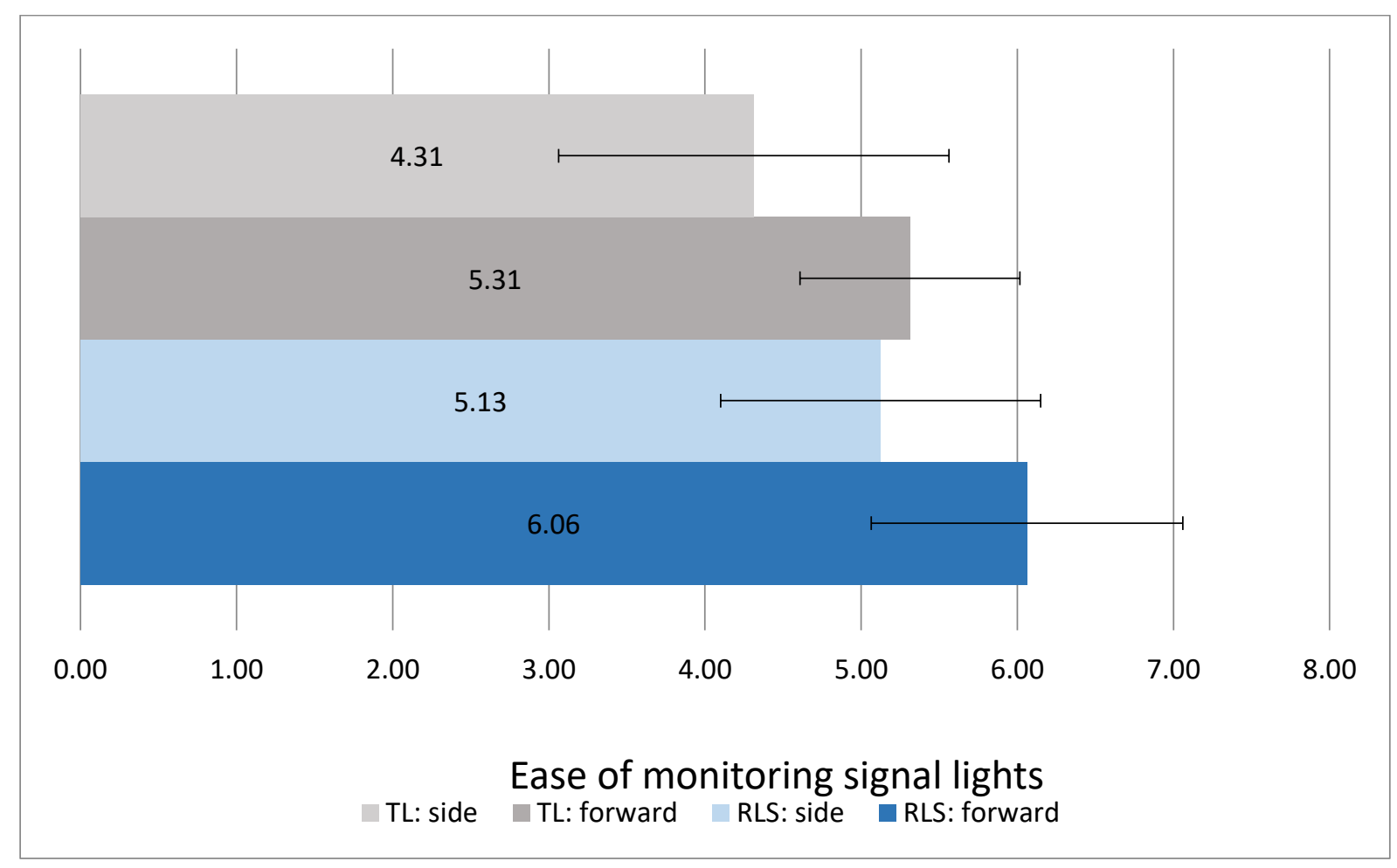

Figure 8 - mean scores for ease of monitoring

\subsection{Level of tiredness}

Another question in the in-between segment interview was related to level of tiredness. A 7-point Likert scale was used, where seven is the least tired and one is the most tired. A two-way repeated measure ANOVA with a Greenhouse-Geisser correction was used to assess the results which revealed no significant effect in the level of tiredness between different light signal type $[F(1,17)=$ $1.824, p=.195]$ or standing position $[F(1,17)=4.014, p=.061]$. The mean scores are shown in figure 9 . 


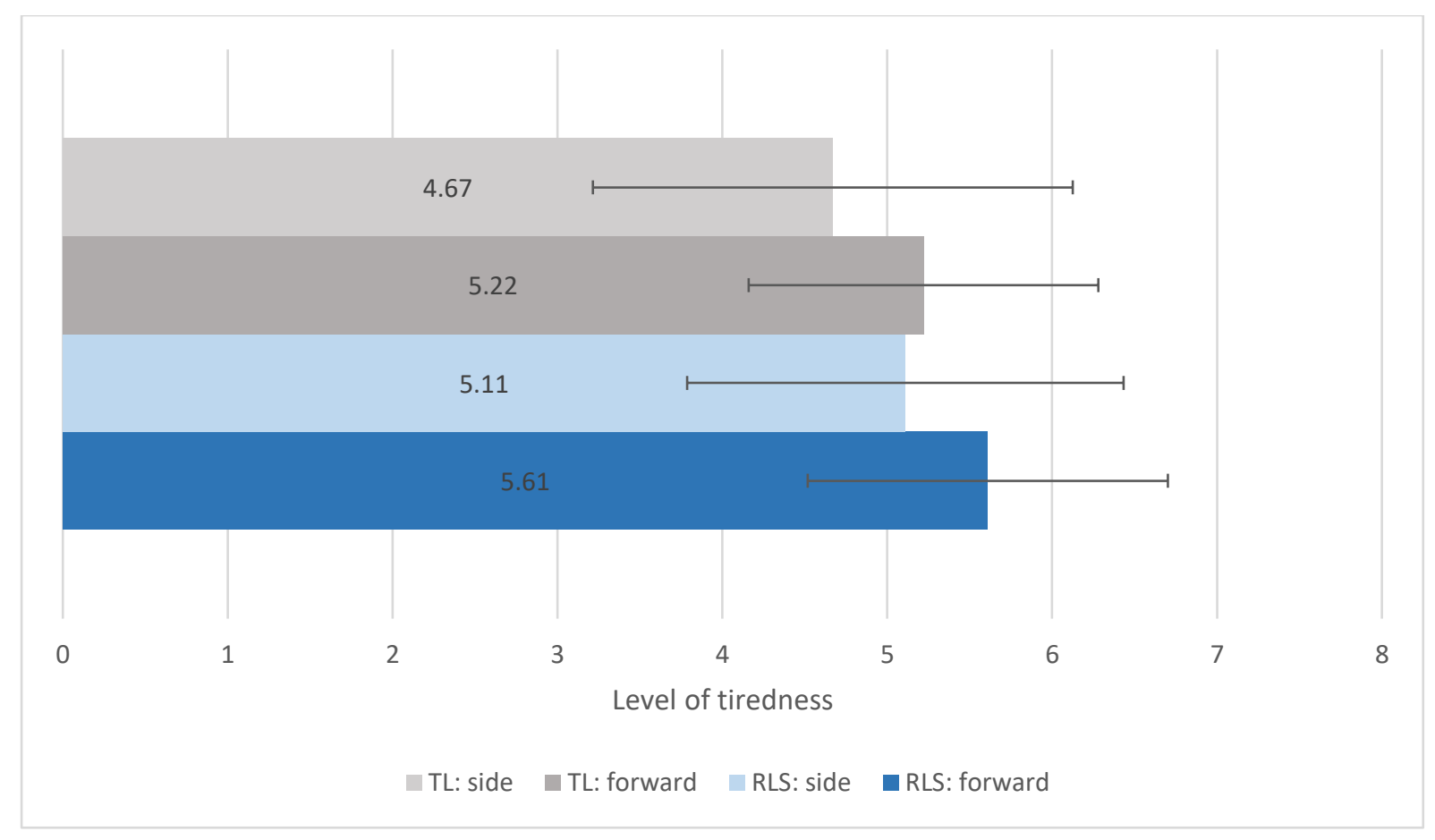

Figure 9 - results of level of tiredness, RLS: side scored higher than TL: side but lower TL: forward due to the additional effort required for the participants to deliberately turn their head and monitor the light source outside of their peripheral

\subsection{Eye tracking results}

Participants wore a pair of eye tracking device throughout the experiment to track their eyes movements in different scenarios. The data were used to create heat maps which illustrate the average fixation time in various AOIs (figure 10). It is observed that in scenarios where participants monitor the tower light source at least $10 \%$ of fixation durations is spent looking at the tower light, which reduce time spent looking at the manual work top where the assembly task is being carried out. 


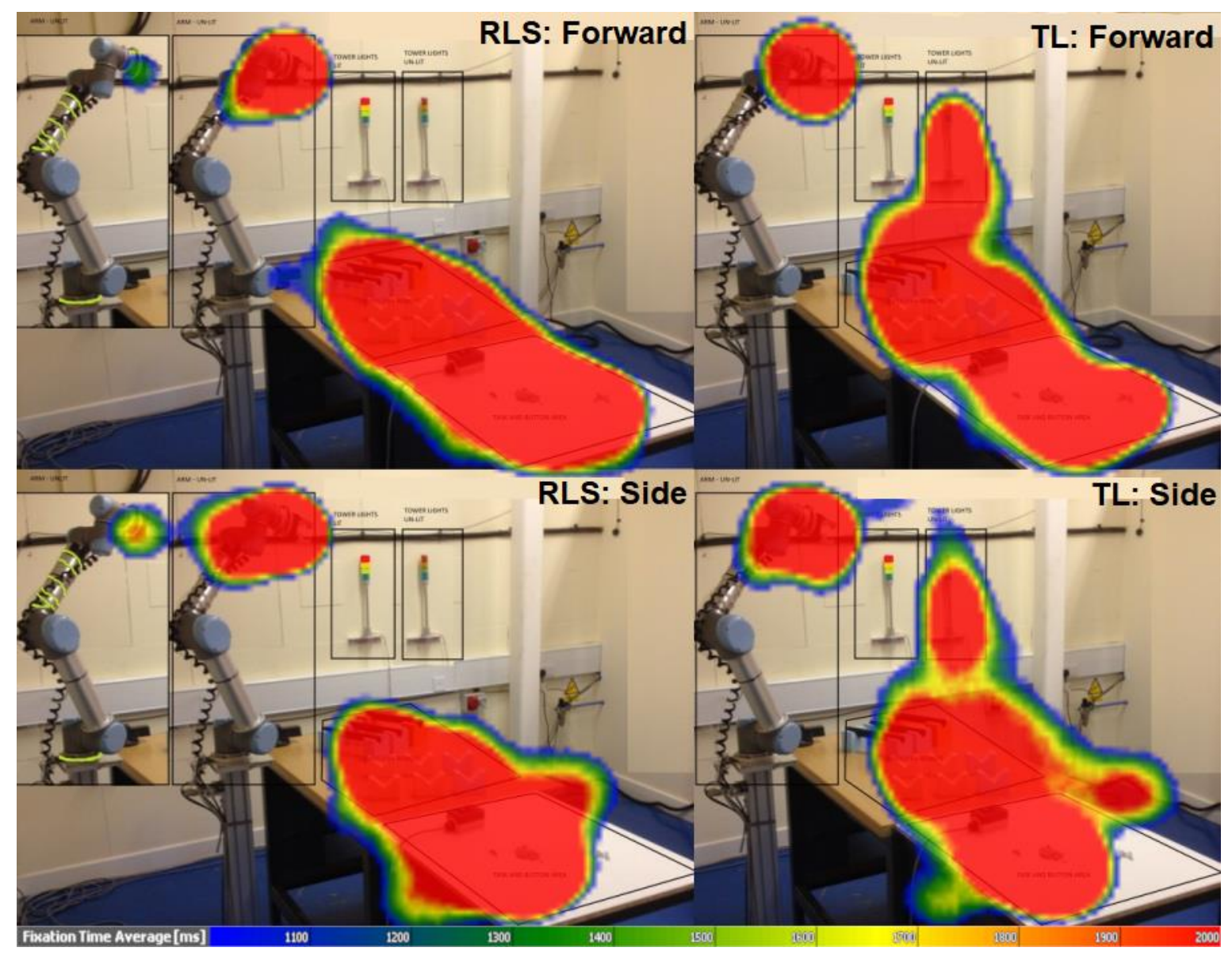

Figure 10 - combined average fixation heat map (1000-2000ms) of the four scenarios, an additional $\mathrm{AOI}$ was added in RLS scenes showing fixations on the robot when lighted

The fixation time average and fixation count average in the manual worktop is higher in the two scenarios with the RLS than those with the tower light source. In number, the fixation time average (manual task bench) of the sideward facing robot light setting is $9 \%$ more than the tower light setting (figure 11) while fixation count is 52.3 times more. In the forward facing scenarios, the robot light setting fixation time average is also $9 \%$ over the tower light setting while fixation count is 53.2 times more. It can be an indication of higher allowance of attention on manual task under the integrated robot light condition. In side standing position, participants had to turn their body or their head in order to see the robot and the pipe bench, so participants generally spend more time looking at the task bench as shown in figure 11 . On the other hand, participants have spent more time paying attention at the pipe bench in the forward standing position. A two-way repeated measure ANOVA with a Greenhouse-Geisser correction was used to assess the eye tracking mean dwell time data which revealed significant effects in the interaction between signal light type and AOIs $[F(2.512,35.169)=6.942, p=.002]$ and significant difference was also found in signal type $[F(1$, $14)=14.882, p=.002]$. The same test was used to analysis the mean fixation time which showed significant differences in the signal types $[F(1,14)=16.76, p=.001]$ and in the interaction between signal type and $A O I s[F(2.322,32.511)=8.519, p=.001]$. 


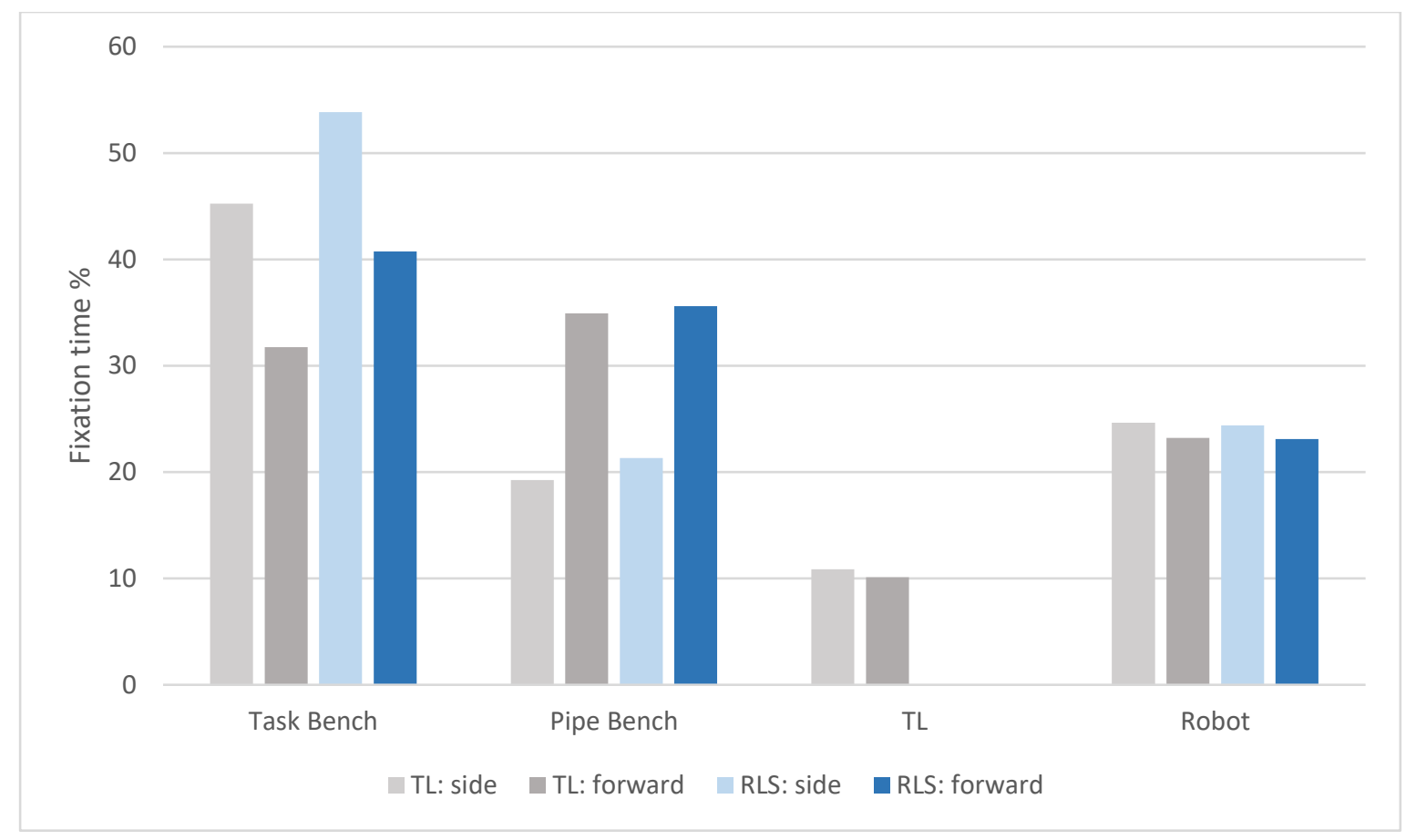

Figure 11 - average fixation time percentage of all participants, participants have spent at least $10 \%$ of time fixated on the tower light in TL scenarios

\subsection{Qualitative results}

Apart from quantitative measurements, semi-structured interviews were used at the end of each experiment. The questions were designed to extract information on user's preference as well as other experimental design related question to enhance future work. One of the question was "How did you find the signal lights? Which one do you prefer? And why?". 12 out of 16 participants preferred the RLS due to its large illumination area which grab their attention when lit. One particular participant found it easier to see the static light tower, but preferred the robot light because it helped to monitor the robot. Similarly three other participants preferred the tower lighter, they found it easier because it was a fixed target. However, only one out of these four participants had faster reaction time when the tower light was used. Another question was "How easy did you find it to observe the robot movement?" where most participants stated it was easy to monitor the robot apart from two participants. Three participants indicated that it was easier to observe robot movements when the RLS was in use.

11 participants have some negative comments regarding the comfort level of wearing Eye Tracker during the experiment where five have stated it was "uncomfortable" to wear. 13 out 16 participants have reported that the Eye Tracker obstruct part of their peripheral view. This is justifiable in this experiment, because it resembled a real industrial scenario where operators are obliged to wear safety glasses for personal protection.

The results from the experiment generally show that the integrated robot indication light has a positive effect when comparing to tower light in the same standing position. Improvements have been shown on the reaction time of participants, less misses of light signals and directs more attention to a manual task and robot arm. It also suggests that the effectiveness of signal light can be affected by its size, position and dynamics. 


\section{Discussion}

An exploratory experiment was carried out to prove the RLS can improve communication in industrial human-robot collaboration in terms of assisting human operators to react faster to signals and focus on their tasks. Participants were asked to react to signal lights while performing a manual assembly task and observing a robot performing a pick and place task. The experiment was setup to resemble real life production scenarios where human operators have to perform manual tasks while observing production machines for signal. It is setup at a baseline level with a relatively simple manual task and minimum deviation in tower light distance.

Participants reacted faster to the RLS than the tower light in general, and the hit-rate of the RLS signals was significantly higher than the tower light signals. It was observed that participants often miss light signals when they are focusing on the nut and bolt assembly task. However, it was also noticed that majority of participants could carry out the manual task without looking at the task since it is a relatively simple task. The results showed no correlation between light signal type and manual task performance which could be an indication that the task only require minimal visual attention. Thus, it is likely that increasing the task difficulty will increase the reaction time, reduce hit-rate or decrease manual task performance. Furthermore, participants generally gave higher score to the RLS in terms of ease of monitoring during the semi-structured interview between each experiment segment. The final questionnaire at the end of each experiment revealed that participants prefer the RLS for its dynamism and larger illuminated area compared to the tower light. It has been previously found that people react quicker to dynamic events compare to static occurrences, which supports the finding in this study [38].

The eye tracking results revealed that participants' attention was diverted to the tower light in the two conditions with the tower light. Participants generally spend less time looking at their manual assembly task when having to react to signals from the tower light than when the signals came from the RLS. This could be explained using the top-down attention theory that when monitoring the static tower light participants knew the location of the tower light and they deliberately searched for it. Whereas the RLS is part of the robot body which does not create a diversion.

\section{Summary and conclusions}

This paper presents a novel solution to improve communication between humans and robots in industrial collaborative applications. A validation experiment has been carried out at baseline level to evaluate the benefit of the proposed system over conventional tower lights. It was hypothesised that the RLS will perform better than the tower light in terms of providing participants with greater awareness and reducing the workload. The results show that the RLS performs better than the tower light in all experimental aspects. Participants reacted faster to the RLS in both scenarios and it is predicted the difference in react time between the two systems will increase when the tower light is placed further away from the robot base. The RLS also exhibits higher hit-rate than the tower light; it is noticed that participants who have spent longer looking at the manual task bench are more likely to miss signals, which mean that using manual tasks with higher difficulty could proliferate the difference in hit-rate. Participants tended to rate the RLS as having less impact on their overall workload and most found it easier to observe due to its size and dynamic nature. Eye tracking results show that participants have more time to spend observing their own task as well as the robot task when the RLS was in use, and when the tower light was in use participants' focus was diverted due to the additional point of interest.

Future study should investigate the effect of the RLS concept on a larger scale. Using a larger industrial robot will increase the distance between the tower light and the moving manipulator 
which could signify the effect of the RLS on operators' reaction time, awareness and performance on the manual task. Furthermore, the comparison should also be carried out in a multi-robot setting similar to those in production environments.

\section{Acknowledgement}

The authors would like to thank their colleagues for constructive criticism of the manuscript. This work was funded by the EPSRC Centre for Innovative Manufacturing in Intelligent Automation under the grant reference number EP/1033467/1.

\section{Reference}

[1] Mitsi, S., Bouzakis, K. D., Mansour, G., Sagris, D., \& Maliaris, G. (2005). Off-line programming of an industrial robot for manufacturing. The International Journal of Advanced Manufacturing Technology, 26(3), 262-267.

[2] Heyer, C. (2010, October). Human-robot interaction and future industrial robotics applications. In Intelligent Robots and Systems (IROS), 2010 IEEE/RSJ International Conference on (pp. 4749-4754). IEEE.

[3] Tan, J. T. C., Duan, F., Zhang, Y., Watanabe, K., Kato, R., \& Arai, T. (2009, October). Humanrobot collaboration in cellular manufacturing: design and development. In Intelligent Robots and Systems, 2009. IROS 2009. IEEE/RSJ International Conference on (pp. 29-34). IEEE.

[4] Imai, M. (1997). Gemba kaizen: a commonsense, low-cost approach to management. McGraw Hill Professional.

[5] Monden, Y. (1983). Toyota production system: practical approach to production management. Engineering \& Management Press.

[6] Greif, M. (1991). The visual factory: building participation through shared information. CRC Press.

[7] Li, J., \& Blumenfeld, D. E. (2005, April). Analysis of andon type transfer production lines: A quantitative approach. In Robotics and Automation, 2005. ICRA 2005. Proceedings of the 2005 IEEE International Conference on (pp. 278-283). IEEE.

[8] Subramaniam, S. K., Husin, S. H., Singh, R., \& Hamidon, A. (2009). Production monitoring system for monitoring the industrial shop floor performance. International journal of systems applications, engineering \& development, 3(1), 28-35.

[9] Baudin, M. (2002). Lean assembly: the nuts and bolts of making assembly operations flow. CRC Press.

[10]Anitsal, i. (2005). Technology-based self-service: From customer productivity toward customer value. University of Tennessee.

[11]Schatz, E. (2003). Scan-it-yourself checkout lines. Wall Street Journal (March 4) D, 2.

[12]Orel, F. D., \& Kara, A. (2014). Supermarket self-checkout service quality, customer satisfaction, and loyalty: Empirical evidence from an emerging market. Journal of Retailing and Consumer Services, 21(2), 118-129.

[13]Boy, G. A. (Ed.). (2012). The handbook of human-machine interaction: a human-centered design approach. Ashgate Publishing, Ltd.

[14]Endsley, M. R. (2011). Designing for situation awareness: An approach to user-centered design. CRC Press.

[15]James, W. (1913). The principles of psychology, Vol II

[16]Katsuki, F., \& Constantinidis, C. (2014). Bottom-up and top-down attention: Different processes and overlapping neural systems. The Neuroscientist, 20(5), 509-521.

[17]Nothdurft, H. (1993), "Saliency effects across dimensions in visual search", Vision research, 33(5), 839-844. 
[18]Taylor, K., \& Stein, J. (1999). Attention, intention and salience in the posterior parietal cortex. Neurocomputing, 26, 901-910.

[19]Cheal, M., Lyon, D. R., \& Hubbard, D. C. (1991). Does attention have different effects on line orientation and line arrangement discrimination?. The Quarterly Journal of Experimental Psychology, 43(4), 825-857.

[20]Connor, C. E., Egeth, H. E., \& Yantis, S. (2004). Visual attention: bottom-up versus topdown. Current Biology, 14(19), R850-R852.

[21]Hein, E., Rolke, B., \& Ulrich, R. (2006). Visual attention and temporal discrimination: Differential effects of automatic and voluntary cueing. Visual Cognition, 13(1), 29-50.

[22] Ling, S., \& Carrasco, M. (2006). Sustained and transient covert attention enhance the signal via different contrast response functions. Vision research, 46(8), 1210-1220.

[23]Liu, T., Stevens, S. T., \& Carrasco, M. (2007). Comparing the time course and efficacy of spatial and feature-based attention. Vision research, 47(1), 108-113.

[24]Müller, H. J., \& Rabbitt, P. M. (1989). Reflexive and voluntary orienting of visual attention: time course of activation and resistance to interruption. Journal of Experimental psychology: Human perception and performance, 15(2), 315.

[25]Pinto, Y., van der Leij, A. R., Sligte, I. G., Lamme, V. A., \& Scholte, H. S. (2013). Bottom-up and top-down attention are independent. Journal of vision, 13(3), 16.

[26]Murray, S. A., \& Caldwell B. S. (1996). Human performance and control of multiple systems. Human Factors, 38, 323-329.

[27]Schneider, D. W., \& Anderson, J. R. (2011). A memory-based model of Hick's law. Cognitive Psychology, 62(3), 193-222.

[28]Simon, J. R., \& Wolf, J. D. (1963). Choice reaction time as a function of angular stimulusresponse correspondence and age. Ergonomics, 6(1), 99-105

[29]Miles, C., Auburn, T. C., \& Jones, D. M. (1984). Effects of loud noise and signal probability on visual vigilance. Ergonomics, 27(8), 855-862.

[30]Warner, H. D., \& Heimstra, N. W. (1973). Target-detection performance as a function of noise intensity and task difficulty. Perceptual and motor skills, 36(2), 439-442.

[31]Soichiando, \& Oda, S. (2001). Central and peripheral visual reaction time of soccer players and nonathletes. Perceptual and Motor Skills, 92(3), 786-794.

[32]Uemura, K., Yamada, M., Nagai, K., Mori, S., \& Ichihashi, N. (2012). Reaction time for peripheral visual field increases during stepping task in older adults. Aging clinical and experimental research, 24(4), 365-369

[33]Michaud, F., \& Vu, M. T. (2001). Light signaling for social interaction with mobile robots. In proceedings of 2001 IEEE International Symposium on Computational Intelligence in Robotics and Automation (pp. 137-142). IEEE.

[34]BRITISH STANDARDS INSTITUTE, 2002. BS EN 60073: Basic and safety principles for manmachine interface, marking and identification. Coding principles for indicators and actuators. London: British Standards Institute.

[35]BRITISH STANDARDS INSTITUTE, 2008. BS EN 61310: Safety of machinery. Indication, marking and actuation. Requirements for visual, acoustic and tactile signals. London: British Standards Institute.

[36]Santner, K., Fritz, G., Paletta, L., \& Mayer, H. (2013, May). Visual recovery of saliency maps from human attention in 3D environments. In 2013 IEEE International Conference on Robotics and Automation (ICRA) (pp. 4297-4303). IEEE

[37]Walters, M. L., Oskoei, M., Syrdal, D. S., \& Dautenhahn, K. (2011, July). A long-term humanrobot proxemic study. In RO-MAN, 2011 IEEE (pp. 137-142). IEEE. 
[38]Franconeri, S. L., \& Simons, D. J. (2005). The dynamic events that capture visual attention: $A$ reply to Abrams and Christ (2005). Attention, Perception, \& Psychophysics, 67(6), 962-966.

[39]Yuan, M. L., Ong, S. K., \& Nee, A. Y. C. (2008). Augmented reality for assembly guidance using a virtual interactive tool. International Journal of Production Research, 46(7), 1745-1767.

[40]Michalos, G., Karagiannis, P., Makris, S., Tokçalar, Ö., \& Chryssolouris, G. (2016). Augmented reality (AR) applications for supporting human-robot interactive cooperation. Procedia CIRP, 41, 370-375.

[41]Mittendorfer, P., \& Cheng, G. (2011). Humanoid multimodal tactile-sensing modules. IEEE Transactions on robotics, 27(3), 401-410.

[42]Oyekan, J. O., Hutabarat, W., Tiwari, A., Grech, R., Aung, M. H., Mariani, M. P., ... \& Dupuis, C. (2019). The effectiveness of virtual environments in developing collaborative strategies between industrial robots and humans. Robotics and Computer-Integrated Manufacturing, 55, 41-54.

[43]Matsas, E., Vosniakos, G. C., \& Batras, D. (2018). Prototyping proactive and adaptive techniques for human-robot collaboration in manufacturing using virtual reality. Robotics and Computer-Integrated Manufacturing, 50, 168-180.

[44]Corrales, J. A., Candelas, F. A., \& Torres, F. (2011). Safe human-robot interaction based on dynamic sphere-swept line bounding volumes. Robotics and Computer-Integrated Manufacturing, 27(1), 177-185. 
The development and evaluation of Robot Light Skin: A novel robot signalling system to improve communication in industrial pÿhuman robot collaboration

\author{
Tang, Gilbert
}

Elsevier

Tang G, Webb P, Thrower J. (2019) The development and evaluation of Robot Light Skin: A pÿnovel robot signalling system to improve communication in industrial human robot collaboration. Robotics and Computer-Integrated Manufacturing, Volume 56, April 2019, pp. 85-94 https://doi.org/10.1016/j.rcim.2018.08.005

Downloaded from Cranfield Library Services E-Repository 